\title{
Algebraic analysis of topological domain-interface defects in crystals J. McKenzie ${ }^{1}$, B. Campbell ${ }^{2}$ \\ ${ }^{1}$ No affiliation given, ${ }^{2}$ Brigham Young University james.mck28@gmail.com
}

\begin{abstract}
When crystals undergo phase transitions involving group-subgroup relationships, distinct regions of the lowsymmetry child structure can possess distinct directions of the order parameter, which are related to one another via broken parent symmetries. The crystallographic community typically refer to these regions as domains. An interfacial boundary where two or more domains meet constitute a topological defect. Because such defects can either strategically or inadvertently influence material properties, it is important to understand what types of topological domain-interface defects (TBIDs) can arise in a given material. We will demonstrate that TBIDs can be algebraically characterized and classified using basic tools from group theory.
\end{abstract}

